\title{
ACOUSTIC WAVE PROPAGATION IN PRESSURE SENSE LINES
}

\author{
Patrick Vitarius, Physics Dept, University of Alabama in Huntsville* \\ Don A. Gregory, PhD, Physics Dept, University of Alabama in Huntsville ${ }^{\dagger}$ \\ John Wiley, Transportation Directorate, NASA Marshall Space Flight Center \\ Valentin Korman, Madison Research Corporation
}

\begin{abstract}
Sense lines are used in pressure measurements to passively transmit information from hostile environments to areas where transducers can be used. The transfer function of a sense line can be used to obtain information about the measured environment from the protected sensor. Several properties of this transfer function are examined, including frequency dependence, Helmholtz resonance, and time of flight delay.
\end{abstract}

\section{INTRODUCTION}

Data collection and analysis of rocket engine tests are critical steps in understanding the physics of propulsion devices that may be used for space exploration. Current sensor technology must be exploited fully and expanded to cover all the classical parameters such as temperature, pressure, and flow rate. Methods must be established to infer such parameters as turbulence and shock that cannot be measured directly. This report will describe several experiments that were performed at the Marshall Space Flight Center, East Test Area, on two different engines. One of the engines was a solid rocket motor and the other was a liquid fuel engine. Neither engine was dedicated to the experiments reported on here, but rather the experiments were added so as to not interrupt scheduled tests.

The research is centered on gathering and analyzing acoustic and vibration data. There were two separate but related reasons for collecting the data. The acoustic experiments were done in preparation for testing a much larger solid rocket motor later in the year and an engine being developed for the NASA Orbital Space Plane (OSP). The testing done over the last few weeks was done on a small experimental engine called the LLT. It has a throat diameter of about 1.5 inches and is made substantially of a laminated material. It is a liquid oxygen/ liquid hydrogen engine. The primary sensors used are model 102A10 manufactured by PCB Corporation, and have a sensitivity of $50 \mathrm{mV} / \mathrm{psi}$ and a maximum pressure measurement of $1000 \mathrm{psi}$. Other sensors used are manufactured by Enderco, model 85108 , having a sensitivity of $158.4 \mathrm{mV} / \mathrm{psi}$ (millivolts per pound per square inch) and a maximum pressure measurement of 2 psi.

The sensors themselves are quite expensive and cannot be sacrificed every test so a method must be used to move the sensor itself away from the hot engine gasses. A substantial part of the research performed was directed toward determining the most efficient sense line to use for the measure of pressure pulses from the engine. This is not a trivial task because the sense line itself has a unique transfer function that will affect some frequencies more than others. The goal was to determine if hollow tubes, liquid filled tubes, or gel filled tubes would best transmit the actual acoustic spectra. A substantial amount of this work was done in the laboratory before the engine tests were done. Classical linear system concepts were utilized in understanding how the sense lines affected the signal detected by the pressure transducer.

A second task involved designing an experiment to detect off-axis thrust from an engine mounted in a test stand. A test of the 24 inch SPTA engine provided a platform for a simple optical technique that could at least detect an off-axis (torque) component. The experiment was quite simple and involved mounting a mirror on the mounting ring that the engine was hard mounted to. A green solid state laser pointer was obtained and used as the source. The laser was mounted a safe distance away from the motor and the beam reflected from the mirror back to a screen. A digital video camera was then used to record the motion of the green laser spot on the screen. The eventual goal is to use several lasers and mirrors and screens so that a true three-dimensional representation of the off-axis thrust can be created. The goal of this first attempt was to discover the problems and solutions for the method so that the technique would be ready for the OSP tests planned for later this year.

The law of reflection can be used to create a device that accurately measures small changes in angle $e^{\ddagger}$. A lowpower visible range laser is aimed at a small mirror mounted on the test article. The beam is reflected and hits a large screen; a video camera is mounted facing the screen in order to record the path taken by the laser 
spot. Both the laser and the camera can be mounted hundreds of feet from the test article; only a small mirror need enter the test area.

For Phase I of this experiment, a green laser pointer was used ( 5 milliwatts, peak wavelength 532 nanometers). The mirror was a first surface gimbaled laboratory mirror mounted onto the thrust structure with epoxy. With an effective dispersion of 0.4 milliradians, the laser spot fit nicely onto the two-inch wide mirror ${ }^{8}$. The mirror was aligned so as to reflect the spot towards a screen staked into the ground in the shade of some pine trees 250 feet from the test engine. The recording device was a digital video camera recording at $\mathbf{3 0}$ frames per second, outfitted with an inexpensive green filter.

\section{MOTIVATION}

\section{Acoustic Sense Lines}

The acoustic phenomena of interest in propulsion applications often occur in harsh environments where heat, high-velocity particulate matter, and volatile chemicals preclude the use of sensitive and expensive sensors. To avoid the compromise between safe distance and useful data range, a sense line may be used to passively transmit information as a conduit. The sense line should either be durable enough to withstand such conditions, or else inexpensive so that it can be replaced periodically.

The variation in parameters at one end of the tube will uniquely determine the condition at the closed end of the tube, where the sensor is to be located. The sense line must have dimensions small enough that it does not affect the parameters of the system it measures. It should be emphasized that a sense line does not automatically transmit temperature, pressure, and other conditions from one end of the tube to the other. Rather, every measurement taken through a sense line must be analyzed in terms of temporal shift and transfer function losses. In addition, any component of the system that alters data introduces potential error into the measurement, and sense lines are no exception. Transfer functions for fluid-filled sense lines fall to zero for high frequencies as this vibrational energy is absorbed by the fluid; any information about these high frequency components is lost. The time delay due to the length of the sense line is a variable quantity that can depend on conditions (e.g., temperature) inside the sense line.

Acoustic resonance is a phenomenon via which certain vibrational frequencies, or modes, are amplified by the resonating structure. In the analysis of the pressure data transmitted through acoustic sense lines, Helmholtz resonance was clearly observed at frequencies corresponding to the lengths of the sense lines.

The resonance condition for a tube opened at one end is

$$
(2 n+1) \frac{v}{4 L}=f \text {, }
$$

where $n$ is an integer index ranging upwards from zero, $f$ is the frequency of the sound, $v$ is the speed of sound in the resonating tube, and $L$ is the length of the tube ${ }^{* *}$.

Time of flight analysis depends on the assumption that the transit time for each of the tubes is the same; the offset in signal onset from one sensor to the next is solely a factor of the position of the open end of the sense line. If this is the case, then it is expected that

$$
v=\frac{\Delta r}{\Delta t},
$$

where $\Delta r$ is the difference in distance from the nozzle for any two sensors, and $\Delta t$ is the time delay between the two sensors ${ }^{\dagger+}$. It is possible to calculate a mean value for $\mathrm{v}$ by considering the linear fit for the various data points plotted with time on the horizontal axis, and radial distance on the vertical axis.

\section{Optical Off-Axis Thrust Measurements}

Axial thrust structures measure displacement along the principle axis and infer thrust on this axis. Most configurations measure off-axis components of thrust poorly, or not at all. A non-invasive, inexpensive technique was desired for measuring these off-axis components of thrust.

A system was developed in which a laser beam is incident on a mirror mounted on the test stand. The laser and mirror were positioned such that the reflected beam struck a Lambertian screen positioned several hundred feet away. Geometry insures that a small change in orientation angle

will lead to large displacements of the order

$$
\Delta x \approx D \Delta \theta,
$$

where $\Delta x$ is the displacement on the screen, and $\Delta \theta$ is the change in orientation angle of the mirror. A more detailed analysis takes into account the incident angle of the laser beam, the orientation of the screen with respect to the laser and other geometric factors to produce a transcendental equation for $\Delta x$ in terms of $\Delta \theta$. 


\section{RESULTS}

\section{Acoustic Sense Line Transfer Functions}

Various fluids were explored for use in sense lines. A fluid was desired that would transmit much of the energy to the transducer with a known transfer function. A laboratory experiment was designed to compare short sense lines filled with water, agarose, and air to a control transducer used to measure the unaltered shock. Agarose is a polysaccharide obtained from agar. The preparation used in this experiment is a suspension of agarose in a boric acid buffer with a gelatinous. consistency ${ }^{\ddagger \ddagger}$.

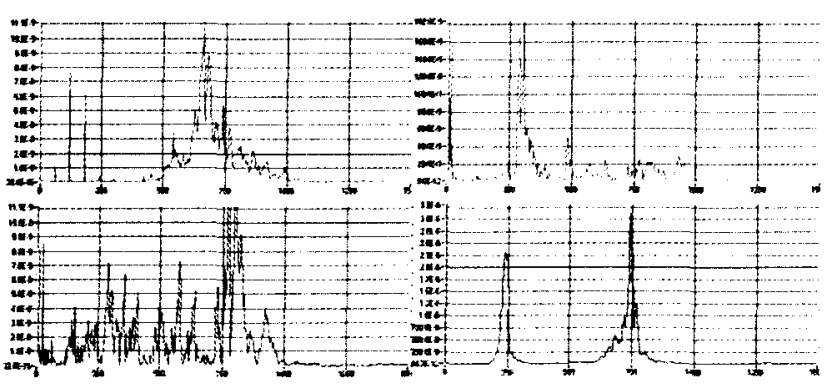

Figure 1: Power spectra of (clockwise from top left): the freestanding transducer, and transducers in sense lines filled with water, air, and an agarose suspension.

The general shape of the transfer functions of the sense lines can be obtained by taking the ratio of each of the power spectra to the power spectrum of the freestanding transducer. This ratio is not the true transfer function, since the power spectra are the squares of Fourier transforms and not the true Fourier transforms, but the general characteristics of the transfer functions should be apparent from such an exercise.

Both water and agarose demonstrate a rough correlation with the freestanding transducer at higher frequencies, in that both mimic the falloff in power at $1000 \mathrm{~Hz}$. But these two sense lines also demonstrate high gain in the lower frequencies that correspond to Helmholtz resonance. This resonance can be seen much more clearly in the air-filled sense line, where the original profile is lost beneath a sharp resonance spike. This resonance is analyzed in more detail in the field tests below.
None of the transfer functions are linear in the sense that no sense line power spectrum differs from the freestanding transducer by a multiplicative constant. All of the sense lines effectively damped out the 60 Hertz (and integer multiple) noise indicated by the sharp, low-frequency spikes in the freestanding transducer.

\section{Acoustic Sense Line Time of Flight Results}

The propagation rate of shock wave can be determined by comparing the onset times for each of the sensors. The onset of the shock due to the combustion event appears as a transient spike followed by a rapid rise to a much higher plateau on each of the channels. As all three sensors were separated from the event by identical lengths of Tygon tubing, the time delay can be attributed to the time of flight of the acoustic wave travelling through the air. A linear fit to these three data points indicated a propagation speed of 1100 feet per second, comparing very favorably to the reference value for acoustic waves, 1086 feet per second at STP. A more extensive array of sensors could detect changes in propagation speed, including regions where the propagation was supersonic, as well as directional difference in propagation speeds.

A qualitative comparison of the signals at each sensor indicates a rather rapid decrease in total energy as distance increase, and a rapid decrease as the sensors are placed further from the axis. A non-directional $\left(1 / r^{2}\right)$ distribution would be independent of direction, whereas a collimated distribution would not show such a rapid falloff with distance ${ }^{\$ 8}$. A more extensive array could determine the shape of the advancing profile by integrating power spectrum of the signal to determine the energy received at each point.

\section{Acoustic Sense Line Helmholtz Resonance}

Helmholtz resonance was clearly observed in the acoustic wave tests. By plotting spectral intensity on a time-frequency plane, broad bands could be observed at regular intervals during the combustion event. Each of these events was indexed with an odd integer corresponding to a harmonic for a closed tube, and the value for $v_{s}$ was used to solve the Helmholtz resonator equation for the length of the tube. A value of 9.5 feet was obtained, very close to the actual length of the tube. 

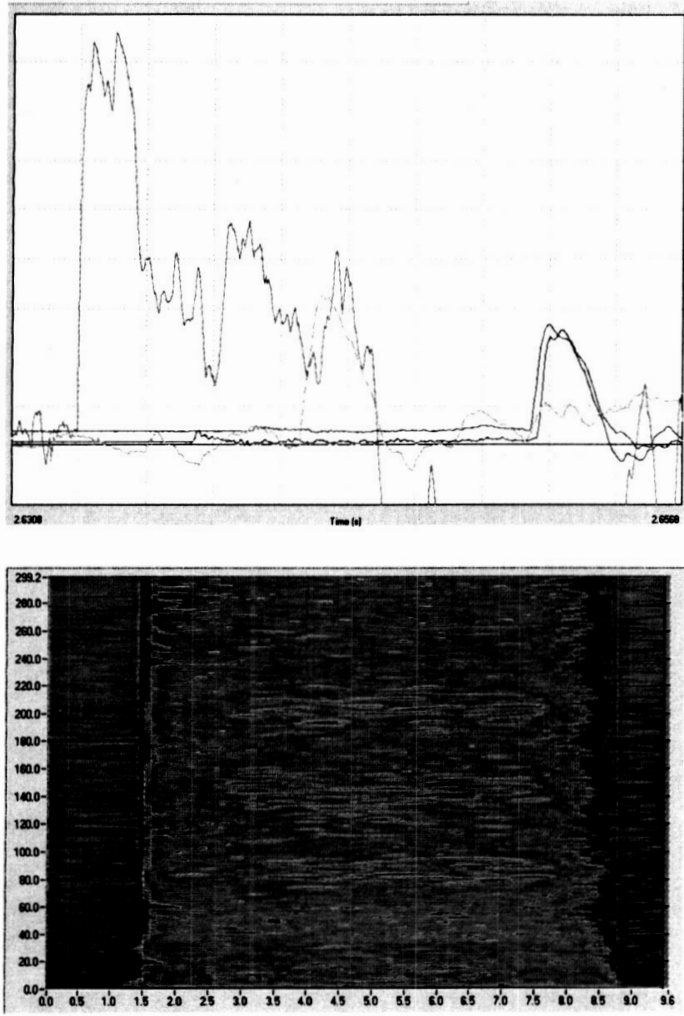

Figure 2: Time-domain data for time of flight measurement; time-frequency plots showing Helmholtz frequencies.

\section{Optical Off-Axis Thrust Measurements}

Phase I demonstrated that the recording device could easily detect the full-intensity spot, and in separate experiments it was shown that small changes in angle resulted in measurable shifts in spot location. It was also proven in the laboratory that the formulae developed for calculating change in angle from these shifts was accurate. However, the logistics of test firing resulted in the laser not operating at full strength during the firing of the motor, and as a result the spot was not visible during that time. It will be necessary in future tests to outfit the laser with a remote switch so that it can be turned on immediately prior to the firing.
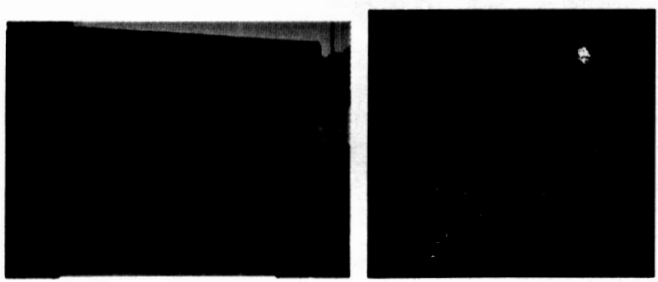

Figure 3: Laser spot on the target screen (left), and an enhanced view of the same image.

\section{CONCLUSION}

Thrust and acoustic measurement procedures were proposed and given initial tests with two rocket motors, one solid and one liquid. The liquid tests showed the validity of using sense lines, and the solid test showed the validity of optical techniques in measuring the off axis torque of engines in test stands. Analysis of the liquid tests confirmed the presence of Helmholtz frequencies, and the time of flight analysis was consistent with a shock wave propagating at sonic speeds. Other measurements are planned for engines later in the year.

${ }^{\ddagger}$ Hecht, E. Optics, Addison Wesley, Second Edition. 1987. (83)

$\S$ Ibid., (422)

${ }^{* *}$ Halliday, D. et al. Fundamentals of Physics, John Wiley and Sons, Fifth Edition. 1997. (436)

${ }^{+\dagger}$ Ibid., (55)

¥ O’Neil, M. (editor) Merck Index: An Encyclopedia of Chemicals, Drugs, \& Biologicals, Merck Publishing Group. 2001. (13, 184)

$\$ \S$ Griffiths, David. Introduction to Electrodynamics, Prentice Hall, Third Edition. 1998. (31) 